\title{
Fast Group Communication Scheduling in Duty-Cycled Multihop Wireless Sensor Networks ${ }^{\star}$
}

\author{
Xiaohua $\mathrm{Xu}^{1}$, Jiannong $\mathrm{Cao}^{2}$, and Peng-Jun $\mathrm{Wan}^{1}$ \\ 1 Illinois Institute of Technology, Chicago IL 60616, USA \\ ${ }^{2}$ Hong Kong Polytechnic University, Kowloon, Hong Kong
}

\begin{abstract}
We study group communication scheduling in duty-cycled multi-hop wireless sensor networks. Assume that time is divided into time-slots and we group multiple consecutive time-slots into periods. Each node can transmit data at any time-slot while it only wakes up at its active time-slot of every period and thus be allowed to receive data. Under the protocol interference model, we investigate four group communication patterns, i.e., broadcast, data aggregation, data gathering, and gossiping. For each pattern, we develop a delay efficient scheduling algorithm which greatly improve the current state-of-the-art algorithm. Additionally, we propose a novel and efficient design to coherently couple the wireless interference requirement and duty cycle requirement.
\end{abstract}

\section{Introduction}

The emergence of wireless sensor networks (WSNs) ushers in a period of prosperous control applications. Contingent on user requests, the control applications usually require efficient delivery of sensory data. The process of streaming data from given source nodes to given destination nodes is termed as group communication. Depending on the number of source nodes and destination (or sink) nodes, there are generally four group communication patterns in WSNs:

Broadcast: a source node sends a common packet to all other nodes.

Data Aggregation: a sink node collects a packet from every other node and every intermediate node combines all received packets with its own packet into a single packet of fixed-size according to some aggregation function such as sum and variance [16].

Data Collection: a sink node collects a raw packet from every other node. Data collection differs from data aggregation in the sense that no in-network processing is allowed for data collection. Thus, each node needs to transmit its raw data and relay all received data towards the sink.

Gossiping: every node broadcasts a packet to all other nodes. Gossiping is also called as all-to-all broadcast in the sense that a gossiping task can be divided into $n$ broadcast task, i.e., each of these $n$ nodes broadcasts its own data once to all other nodes. Here $n$ is the number of nodes in the network. From another viewpoint, a gossiping task consists

\footnotetext{
* This work was supported in part by the National Science Foundation of USA under grant CNS0916666 and by National Natural Science Foundation of P. R. China under grant 61128005.
}

X. Wang et al. (Eds.): WASA 2012, LNCS 7405, pp. 197-205, 2012.

(C) Springer-Verlag Berlin Heidelberg 2012 
of $n$ data collection tasks, i.e., each of these $n$ nodes collects a raw packet from every other node.

The group communication tasks often come with stringent delay constraints imposed by control applications. Here, the delay (or latency) for finishing a group communication task can be interpreted as the duration from the time when the first node transmits for this task, to the time when the destination node(s) receive all required data for this task. One promising way of minimizing the delay is to maximize the network throughput which is subject to the intrinsic wireless interference. A well accepted wireless interference model is the protocol interference model [5], which can serve as a useful abstraction of WSNs. A number of protocols have been proposed in the literature for group communication scheduling such as [2, 4, 6, 10, 16, 18, 20]. However, those work omit the practical duty-cycling scenario where wireless nodes switch between the active state and the dormant state. In this work, we will study delay efficient group communication scheduling in duty-cycled WSNs under the protocol interference model. For each group communication task, we will develop a fast scheduling algorithm and prove that it can achieve an approximation bound that greatly improves the previous best result. Let $P$ be the period of the given duty-cycled network, i.e., the length of time it takes for the wireless nodes to go through a complete on/off cycle, we have

- For broadcast scheduling, we can achieve an approximation bound on latency of $(1+o(1)) \cdot|P|$ while the previous best approximation bound is $17|P|$ in [11].

- For data aggregation scheduling, we can achieve an upper bound on latency of $(6 \Delta+3 R+O(\log R) \cdot|P|$ while the previous best upper bound is $(\Delta+15 R-3) \cdot|P|$ in [12]. Here $R$ and $\Delta$ are the graph radius and the maximum node degree of the communication graph respectively.

- For data collection scheduling, we can achieve an approximation ratio of $10|P|$.

- For gossiping, we can achieve an approximation bound of $10|P|$.

For any uncoordinated duty-cycled WSN, if all nodes's active time-slots in a period are the same (the network has an utilization of at most $\frac{1}{|P|}$ ), each transmission costs one period. In this case, the lower-bound on the delay of finishing any group communication task is $\Omega(|P|)$. However, if nodes's active time-slots in a period are not random, we may improve the network performances by determining each node's active time-slot in advance based on its geographical locations.

The rest of the paper is organized as follows. Section 2 formulates the group communication scheduling problem in WSNs. In Section 3, we construct a universal routing tree for group communication scheduling. Section 4, 5, 6, and 7 are devoted to scheduling algorithm design for broadcast, data aggregation, data collection, and gossiping respectively. We present an energy-efficient unified framework for coupling the addressing of wireless interference and duty-cycling requirement in Section 8 . We review the related results in Section 9 and conclude the paper in Section 10.

\section{Network Model}

Consider a WSN modeled as a communication graph $G=(V, E)$ in a two-dimensional Euclidean plane, where $V$ is the set of all sensor nodes and $E$ is the set of communication links. A node $u \in V$ can transmit data to another node $v \in V$ if $v$ lies within 
the transmission range of node $u$. We assume uniform transmission range normalized to one. Thus, the communication graph turns out to be a unit disk graph $G$, i.e., a communication link exists between any pair of nodes if and only if their distance is at most one. We will focus on the Protocol Interference Model (PrIM) [5]: each node $u$ has an interference range $\rho$ such that any node $v$ will be interfered by the signal from $u$ if $\|u v\| \leq \rho$ and node $v$ is not the intended receiver of the transmission from $u$. Similar to most of the existing work such as [16], [20], we assume that $\rho=1$ for simplicity.

In a duty-cycled scenario, time is divided into time-slots and every consecutive $|P|$ time-slots are grouped into a period. Each node chooses one active time-slot in $P$ randomly and independently. Each node can transmit data at any time-slot while it only wakes up at its active time-slot of every period and thus be allowed to receive data.

Given a group communication task (i.e., broadcast, aggregation, collection, or gossiping) under this duty-cycled scenario, the objective is to design routing and a transmission schedule to finish this task with minimum delay. Here the transmission schedule specify the node activities and can be interpreted as an assignment of the transmission time-slots to all nodes, i.e., a mapping $V \mapsto 2^{N}$. Then a valid communication schedule in $G(V, E)$ with delay $L$ can be defined as a sequence of sender sets $S_{1}, S_{2}, \cdots, S_{L}$ such that if all nodes transmit according to this sequence, this group communication task can be finished successfully. The scheduling problem for broadcast, data aggregation and gossiping in the duty-cycled scenario has been proven to be NP-hard in [11], [12] and [14] respectively.

\section{Routing Tree}

The construction of routing tree relies on a concept of Connected Dominating Set (CDS). Please refer to a recent survey [1] and references therein on CDS. In a graph $G=(V, E)$, a subset $V^{\prime}$ of $V$ is a dominating set (DS) if each node in $V$ is either in $V^{\prime}$ or adjacent to some node in $V^{\prime}$. Nodes in $V^{\prime}$ are called dominators, whereas nodes not in $V^{\prime}$ are called dominatees. A subset $C$ of $V$ is a CDS if $C$ is a dominating set and $C$ induces a connected subgraph.

The routing tree rooted at a node $u$ is constructed as follows. Starting from $u$, we perform breadth-first-search $(B F S)$ over $G$ to build the BFS tree $T_{B F S}$; We next select the maximal independent set (MIS) of $T_{B F S}$ by an existing approach [15], and use this MIS as dominating set; We connect MIS by using some nodes (i.e. connectors) to form a CDS $G_{c}$ of $G$; For each dominatee $v$ not in $G_{c}$, we connect it to $G_{c}$ by adding a link from $v$ to one of its neighboring dominators. The about operations result in a routing tree $T$. It is easy to verify that the depth of $T$ is at most $R_{u}+1$, where $R_{u}$ is the hop distance between the root $u$ and and its farthest node.

We will associate the tree $T$ with ranks of the nodes by using the method in [9, 16]. We proceed ranking layer-by-layer in the bottom-up manner. Initially, $\operatorname{rank}(v)=0$ for each node $v$ in the bottom layer. For each layer $i$ from $R$ down to 1 , for each node $u$ in level $i$, we assign the ranks as follows: if $u$ has no child, $\operatorname{rank}(u)$ is set to zero; if $u$ has at least one child, let $r$ be the maximum rank of its children. If $u$ has only one child of rank $r$, then $\operatorname{rank}(u)$ is set to $r$; otherwise $\operatorname{rank}(u)$ is set to $r+1$. As observed in [9, 16], each node has rank no more than its parent in $T$, and for each node $v \in V$, we have $\operatorname{rank}(v)=O(\log |V|)$. 


\section{Broadcast Scheduling}

We first construct a routing tree rooted at the source node and ranks of nodes. Then, the broadcast scheduling process consists of two phases: (1) broadcast data to all nodes in the CDS, (2) broadcast data from dominators to dominatees.

In the first phase, for each integer $0 \leq i<R$ (node level) and $0 \leq j<r$ (rank) where $R$ is the depth of the routing tree and $r$ is the rank of the distinguished source node, set $V_{i j}$ to be the set of nodes in layer $i$ with rank $j$, and $V_{i j}^{\prime}$ to be the set of their children. We perform scheduling for the pair of node subsets $\left(V_{i j}, V_{i j}^{\prime}\right)$ as follows. Let $W_{0}$ be the set of parents of nodes in $V_{i j}^{\prime}$ with rank $j$. We apply the algorithm ILBS in [9] to generate a $\left(V_{i j}, V_{i j}^{\prime} \backslash \operatorname{In} f\left(W_{0}\right)\right)$-schedule $\left(W_{1}, W_{2}, \cdots, W_{16}\right)$ where $\operatorname{In} f\left(W_{0}\right)$ is the set of nodes in $V \backslash W_{0}$ each of which has exactly one neighbor in $W_{0}$. Then, for each $0 \leq k \leq 16$, all nodes in $W_{k}$ transmit in the $(i+51(r-j)+3 k)$-th period when their children are awake.

In the second phase, we schedule the transmissions by the dominators as follows: partition the set $V^{\prime}$ of dominators into 2-independent sets $\left\{V_{i}^{\prime}: 1 \leq i \leq 12\right\}$ [9]. Here 2 -independent set means that the pairwise distance of nodes in this set are all greater than 2. The dominators in $V_{i}^{\prime}$ transmit in the $i$-th period when their children are awake.

Next, we analyze the performance of our broadcast scheduling algorithm.

Theorem 1. The proposed algorithm for broadcast scheduling produces a correct broadcast schedule of latency $(R+\log R)|P|$ where $R$ is the graph radius of the communication graph and $P$ is a period in a duty-cycled network.

Proof. The correctness proof involves two arguments: (1) any pair of concurrent transmitting nodes will not conflict with each other, (2) any node will receive the data before it transmits. Both arguments are similar to [9].

In the first phase, each node in the CDS needs $|P|$ to transmit its message to all the children, thus this phase costs at most $(R+51 r)|P|$. By the folklore area argument, we have that the size of the CDS is bounded by $O\left(R^{2}\right)$. This implies that $r=O(\log R)$. Thus, this phase has latency at most $(R+O(\log R)) \cdot|P|$. In the second phase, the broadcast schedule takes at most $12|P|$ time-slots. Thus, the theorem follows.

The lower bound for the broadcast scheduling problem is $R$. The lower bound can be achieved when the network topology is a chain and the active time-slot of any node is exactly one time-slot after that of its parent in the routing tree. Thus, we have the following theorem.

Theorem 2. The proposed algorithm for broadcast scheduling can achieve $(1+o(1))$. $|P|$-approximation bound where $P$ is a period in a duty-cycled network.

\section{Data Aggregation Scheduling}

We first construct a routing tree (inward-arborescence) rooted at the sink node and ranks of nodes. Then, the data aggregation scheduling process consists of two phases: (1) dominatees transmit data to dominators, (2) dominators route data towards the root. 
The first phase consists of one-hop data transmissions from dominatees to dominators. We will split this phase into multiple rounds. In each round, for every dominator $u$, we first select a link $\overrightarrow{v u}$ from one of its neighboring dominatees to itself. Assume all selected links form a set $\mathcal{L}$. We then transmit links in $\mathcal{L}$. It is easy to verify that each round costs at most $6|P|$ time-slots, where $P$ is the period. Since each dominator has at most $\Delta$ neighboring dominatees, there are at most $\Delta$ rounds. Thus, the first phase costs at most $6 \Delta \cdot|P|$ time-slots.

In the second phase, we will perform data transmissions in a pipelined manner. For each node $u$, let $i$ be its level, let $j$ be its rank, and let $a$ be its active time-slot in a period, $l$ is the label received by the link $\overrightarrow{u v}$ by the algorithm IMC in [16]. If $u$ is a dominator, we will assign a time-slot of $2((R-i)+44 j+4(l-1)) \cdot|P|+a$ to transmit; If $u$ is connector, we will assign a time-slot of $2((R-i)+44 j+4(l-1)) \cdot|P|+|P|+a$ to transmit. In our method, all dominators transmit at even periods only, and all connectors transmit at odd periods only; the time-disjoint property can avoid conflicts between nodes from different groups. It is easy to prove that our method can avoid interference (similar to [16]) and this phase costs at most $(2 R+O(\log R)) \cdot|P|$ time-slots.

Theorem 3. The proposed algorithm for data aggregation scheduling produces an aggregation schedule of latency at most $(6 \Delta+3 R+O(\log R)) \cdot|P|$.

\section{Data Collection Scheduling}

We first construct a routing tree $T$ rooted at the sink node. Our algorithm then relies on a labeling of the edges of $T$. We first order all nodes in $V$ in the decreasing order of depth in $T$. Ties are broken arbitrarily. Assume the resulting sequence is:

$$
\sigma \longleftarrow<v_{1}, v_{2}, \cdots, v_{n}>
$$

We assign the $j$-th edge in the tree path from the sink node to $v_{i}$ with a label $2(i-1)+j$. For an edge connecting $v_{i}$ and its parent, the number of assigned labels is equal to the number of descendants of $v_{i}$ in $T$. If $v_{i}$ is connector (dominator), all labels received by the edge between $v_{i}$ and its parent are odd (even). The edges across two consecutive layers of the dominating tree receive distinct labels. Considering a node $v_{i}$, let $h$ be the length of the path from the sink node to $v_{i}$. The maximum label assigned to the edges in the path from the sink node to $v_{i}$ is $2(i-1)+h$. It is sufficient to show that $2(i-1)+h \leq 2 n-3$, i.e., the largest label is $2 n-3$.

For each number $k$, let $E_{k}$ denote the set of edges of $T$ which contains a label $k$, and $A_{k}$ denote the links in the inward $s$-arborescence oriented from the edges in $E_{k}$. Here $s$ denotes the sink node. Then, if $k$ is odd (respectively, even), all the receiving (respectively, transmitting) endpoints of links in $A_{k}$ are dominators. Moreover, every dominator is incident to at most one link in $A_{k}$.

Then, the transmission schedule are partitioned into $2 n-3$ rounds. The $k$-th round $(1 \leq k \leq 2 n-3)$ is scheduled as follows. We color all dominators such that for each non-dominating node, all its one-hop neighboring dominators receive distinct colors. Since at most 5 dominators can be adjacent to a common node, the number of colors used is at most 5 . For each link, assume its dominator endpoint receives the $i$-th color 
and the active time-slot for duty cycling of the corresponding sender is $a$, then this link is scheduled in the $i \cdot|P|+a$-th time-slot of the $k$-th round. Thus, each round takes at most $5|P|$ time-slots. It is easy to verify the correctness of this transmission schedule, in addition, the schedule has a delay of at most $5(2 n-3) \cdot|P|$.

As $n-1$ is a trivial lower bound on the delay for data collection, the approximation ratio of the transmission schedule presented is at most $10|P|$.

Theorem 4. The proposed scheduling algorithm for data collection achieves an approximation bound of $10|P|$.

Unbounded Packet-Size Model: Since the packet-size can be arbitrarily large, we can adopt our transmission schedule for data aggregation (described in Section 5 to finish this data collection task under the unbounded packet-size model.

\section{Data Gossiping Scheduling}

Let the graph center of the network be a node $v_{c}$ such that the hop distance of the path from node $v_{c}$ to the node farthest from $v_{c}$ in the network reaches minimum. We will use $v_{c}$ as the root to construct a routing tree. The gossiping scheduling process consists of two phases. In the first phase, the graph center $v_{c}$ collects data from all the other nodes. We will apply our transmission scheduling for data collection presented in Section 6 In the second phase, the graph center broadcasts all data one by one to all other nodes.

We first color all dominators such that for each non-dominating node, all its one-hop neighboring dominators receive distinct colors. The coloring can ensure that all dominators with the monotone color can transmit (or receive) data concurrently without conflict. Since at most 5 dominators can be adjacent to a common node, the number of colors used is at most 5 . By proper renumbering of the colors, we assume that $v_{c}$ has the first color. We group the time-slots into $10|P|$-slot frames. In each frame, the first $5|P|$ slots form a dominator sub-frame, and the remaining $5|P|$ slots form a connector subframe. Only dominators (respectively, connectors) are allowed to transmit in the dominator (respectively, connector) subframe in each frame. The node $v_{c}$ transmits one packet in each frame. Upon receiving a packet in a dominator subframe, for each connector, assume the color of its child dominator is $i(0 \leq i<5)$, assume the active time-slot of its child dominator is $i$ in a period is $a$, this connector transmits the received packet in the time-slot of $(5+i) \cdot|P|+a$; clearly, this transmission occurs in a connector subframe. Upon receiving a packet in a connector subframe, for each dominator with color $i(0 \leq i<5)$, assume the active time-slot of its child dominator is $i$ in a period is $a$, then this dominator transmits in the time-slot of $i \cdot|P|+a$. Clearly, this transmission occurs in a dominator subframe.

It is easy to verify the correctness of the above schedule. We then bound the delay of the second phase. After $n-1$ frames, $v_{c}$ transmits the last packet. After another $R+1$ frames where $R$ is the graph radius, the last packet reaches all nodes. Therefore, the total number of time-slots takes by the second phase is at most

$$
10|P| \cdot((n-1)+(R+1))=10(n+R) \cdot|P| .
$$

The first phase takes at most $5(2 n-3) \cdot|P|$ time-slots. Hence, the total number of time-slots taken by the two phases is at most 


$$
10(n+R)|P|+5(2 n-3) \cdot|P|=(20 n+10 R-15) \cdot|P| .
$$

Theorem 5. The proposed scheduling algorithm for gossiping can achieve an upper bound on delay of $(20 n+10 R-15) \cdot|P|$.

Clearly, the minimum gossiping latency of $G$ is at least $n-1+R$ where $R$ is the graph radius of $G$, thus we have:

Theorem 6. The proposed scheduling algorithm for gossiping can achieve an approximation bound of $20|P|$.

Unbounded Packet-Size Model: Under this model, we can just perform broadcast only once instead of $n$ times where $n$ is the number of nodes. The broadcast phase, the graph center will transmit the combined data of the packets from all nodes only once.

\section{Coupling Duty Cycle Requirement and Interference Requirement}

In Section 1, we have showed that for any uncoordinated wireless networks, if all nodes's active time-slots in a period are the same, then the network has an utilization of at most $\frac{1}{|P|}$. Thus, when $|P|$ becomes large, the duty cycle requirement will cause the network performance to be very bad. On the other hand, wireless interference requirement also limits the network performance. We will propose a novel design that can address both challenges simultaneously and greatly improve the network performances such as energy-efficiency and delay. Our design will couple interference requirement and duty cycle requirement tightly.

Let $|P|$ be the length of a period. Let $K=\lceil\sqrt{|P|}$. We will employ a grid partition of the deployment plane. The vertical lines $x=i \cdot \ell$ for $i \in \mathbb{Z}$ and horizontal lines $y=j \cdot \ell$ for $j \in \mathbb{Z}$ partition the planes into half-open and half-closed grids of side $\ell$ (here $\mathbb{Z}$ represents the integer set):

$$
\{[i \ell,(i+1) \ell) \times[j \ell,(j+1) \ell): i, j \in \mathbb{Z})\} .
$$

Next, we color the grids such that one grid among every $K^{2}$ grids is assigned with the same color. Each node will determine its active time-slot of a duty-cycled scheduling period based on its geographical locations. At the same time, if at most one node from every grid with a monotone color transmits simultaneously, the transmissions are interference-free subject to the wireless interference requirement.

We then index the colors and denote $\sigma_{g}$ as the color of grid $g\left(\sigma_{g} \in\left\{0,1, \cdots, K^{2}-\right.\right.$ $1\})$. Note that a number of methods have been proposed in the literature to approximate the geometric locations of nodes. For each node $u \in V$, let $\sigma_{g}$ be the color index of the grid $g$ where $p$ lies, we then assign $\sigma_{g}$ as an active time-slot in each duty cycle period for this node $u$. For each of the above group communications, we can perform a partition-based scheduling and the delay will be greatly reduced. 


\section{Related Work}

We first conduct literature review on delay efficient group communication scheduling in WSNs. For broadcast scheduling, Chlamtac and Kutten [3] proved the NP-hardness of finding a minimum makespan collision-free broadcast scheduling for general graphs, even in the absence of wireless interference. Gandhi et al. [4] studied the broadcast scheduling problem for multi-hop wireless networks with bounded transmission range. They present a simple algorithm which guarantees the makespan and the number of retransmissions to be within 468 times their respective optimal values. Huanget al. [9] proposed three approximation algorithms for the broadcast scheduling problem.

Data Aggregation/Collection Scheduling with minimum delay has been proven to be NP-hard [2] and well studied in [10, 16, 18,-20].

Huang et al. [7], [8] studied minimum-latency gossiping in multi-hop wireless networks. Wan et al. [17] studied gossiping scheduling under the approximated physical interference model. Jiao et al. [13,14] studied minimum-latency gossiping in duty-cycle wireless networks.

There are some most recent work on group communication scheduling in uncoordinated duty-cycled wireless networks such as [11] for broadcast scheduling, [12] for data aggregation scheduling and [14] for gossiping scheduling in the duty-cycled scenario respectively.

\section{Conclusion}

We proposed a suite of efficient scheduling algorithms for fast group communication in duty-cycled multi-hop wireless networks under the protocol interference model. The proposed algorithms can achieve the best constant approximation bounds compared to the existing work. We also proposed a novel design to coherently couple the interference requirement and duty cycle requirement which can greatly improve the network performance. As future work, it will be interesting to modify our scheduling algorithms to some other wireless interference models.

\section{References}

1. Blum, J., Ding, M., Thaeler, A., Cheng, X.: Connected dominating set in sensor networks and manets. In: Handbook of Combinatorial Optimization, pp. 329-369 (2005)

2. Chen, X., Hu, X., Zhu, J.: Minimum Data Aggregation Time Problem in Wireless Sensor Networks. In: Jia, X., Wu, J., He, Y. (eds.) MSN 2005. LNCS, vol. 3794, pp. 133-142. Springer, Heidelberg (2005)

3. Chlamtac, I., Kutten, S.: On broadcasting in radio networks-problem analysis and protocol design. IEEE Transactions on Communications, 1240-1246 (2002)

4. Gandhi, R., Mishra, A., Parthasarathy, S.: Minimizing broadcast latency and redundancy in ad hoc networks. IEEE/ACM Transactions on Networking, 840-851 (2008)

5. Gupta, P., Kumar, P.: The capacity of wireless networks. IEEE Transactions on Information Theory 46(2), 388-404 (2000)

6. Hong, J., Cao, J., Li, W., Lu, S., Chen, D.: Sleeping schedule-aware minimum latency broadcast in wireless ad hoc networks. In: IEEE ICC, pp. 1-5. IEEE (2009) 
7. Huang, S., Du, H., Park, E.: Minimum-latency gossiping in multi-hop wireless networks. In: ACM MobiHoc, pp. 323-330 (2008)

8. Huang, S., Wan, P., Du, H., Park, E.: Minimum latency gossiping in radio networks. IEEE TPDS, 790-800 (2010)

9. Huang, S., Wan, P., Jia, X., Du, H., Shang, W.: Minimum-latency broadcast scheduling in wireless ad hoc networks. In: IEEE INFOCOM, pp. 733-739 (2007)

10. Huang, S., Wan, P., Vu, C., Li, Y., Yao, F.: Nearly Constant Approximation for Data Aggregation Scheduling in Wireless Sensor Networks. In: IEEE INFOCOM, pp. 366-372 (2007)

11. Jiao, X., Lou, W., Ma, J., Cao, J., Wang, X., Zhou, X.: Duty-cycle-aware minimum latency broadcast scheduling in multi-hop wireless networks. In: IEEE ICDCS, pp. 754-763 (2010)

12. Jiao, X., Lou, W., Wang, X., Cao, J., Xu, M., Zhou, X.: Data aggregation scheduling in uncoordinated duty-cycled wireless sensor networks under protocol interference model. In: Ad Hoc and Sensor Wireless Networks

13. Jiao, X., Lou, W., Wang, X., Ma, J., Cao, J., Zhou, X.: Interference-Aware Gossiping Scheduling in Uncoordinated Duty-Cycled Multi-hop Wireless Networks. In: Pandurangan, G., Anil Kumar, V.S., Ming, G., Liu, Y., Li, Y. (eds.) WASA 2010. LNCS, vol. 6221, pp. 192-202. Springer, Heidelberg (2010)

14. Jiao, X., Lou, W., Wang, X., Ma, J., Cao, J., Zhou, X.: On interference-aware gossiping in uncoordinated duty-cycled multi-hop wireless networks. In: Ad Hoc Networks (2011)

15. Wan, P.-J., Alzoubi, K.M., Frieder, O.: Distributed construction of connected dominating set in wireless ad hoc networks. In: IEEE INFOCOM (2002)

16. Wan, P., Huang, S., Wang, L., Wan, Z., Jia, X.: Minimum-latency aggregation scheduling in multihop wireless networks. In: ACM MobiHoc, pp. 185-194 (2009)

17. Wan, P., Wang, L., Frieder, O.: Fast group communications in multihop wireless networks subject to physical interference. In: IEEE MASS, pp. 526-533 (2009)

18. Xu, X., Li, X.Y., Mao, X., Tang, S., Wang, S.: An improved approximation algorithm for data aggregation in multi-hop wireless sensor networks. IEEE TPDS (2011)

19. Xu, X., Li, X.Y., Wan, P.J., Tang, S.: Efficient Scheduling for Periodic Aggregation Queries in Multihop Sensor Networks. To Appear in IEEE/ACM Transactions on Networking

20. Yu, B., Li, J., Li, Y.: Distributed Data Aggregation Scheduling in Wireless Sensor Networks. In: INFOCOM (2009) 